\title{
Healthcare professional awareness of eating disorders in people with type 1 diabetes: a staff survey
}

\author{
SARAH BREWSTER, ${ }^{1}$ HELEN PARTRIDGE, ${ }^{2}$ CAROLINE CROSS, ${ }^{3}$ HERMIONE PRICE ${ }^{1}$
}

\begin{abstract}
Background: People with type 1 diabetes are at increased risk of eating disorders. 'Diabulimia', 'Syndrome of Insulin Omission' and 'Type 1 diabetes and Disordered Eating' (T1DE) are all terms used to describe the omission or restriction of insulin in type 1 diabetes for fear of weight gain. For consistency, Type 1 diabetes and Disordered Eating (T1DE) is used throughout this article. The condition is associated with a significant increase in morbidity and mortality but is not yet recognised as a unique entity.

Aim: To determine healthcare professional awareness and knowledge of eating disorders in type 1 diabetes.

Methods: A short, cross-sectional, online healthcare professional survey across primary, secondary and community settings.

Results: Healthcare professional confidence is low when it comes to recognising and supporting people with T1DE, but there is an interest in learning more.

Conclusion: The findings from this study support strategies to improve healthcare professional awareness of T1DE.

Br J Diabetes 2020;20:122-129
\end{abstract}

Key words: type 1 diabetes, eating disorders, diabulimia, insulin omission, healthcare professionals

\section{Introduction}

The careful attention to diet and lifestyle required to manage type 1 diabetes puts individuals at increased risk of developing eating disorders. ' 'Diabulimia', 'Syndrome of Insulin Omission' (SIO) and 'Type 1 Diabetes and Eating Disorder' (T1DE) are used to describe the practice of insulin restriction or omission for fear

Research and Development, Tom Rudd Unit, Moorgreen Hospital, Southern Health NHS Foundation Trust, Southampton, UK

BDEC Department, The Royal Bournemouth and Christchurch Hospitals NHS Foundation Trust, Bournemouth, UK

Dorset HealthCare University NHS Foundation Trust, Poole, UK

Address for correspondence: Dr Sarah Brewster Research and Development, Tom Rudd Unit, Moorgreen Hospital, Southern Health NHS Foundation Trust, Southampton, SO30 3JB, UK E-mail: Sarah.brewster@southernhealth.nhs.uk

https://doi.org/10.15277/bjd.2020.268 of weight gain in people with type 1 diabetes. For continuity we will refer to T1DE throughout the rest of this article.

The prevalence of T1DE is difficult to quantify. Studies have demonstrated an increasing prevalence with age rising from $1 \%$ in pre-adolescence ${ }^{2}$ to $27-39 \%$ in late adolescence and early adulthood. ${ }^{3-5}$ Most screening tools suited to the general population do not accurately detect the presence of disordered eating in people with type 1 diabetes as they focus on behaviours that are inherent to diabetes management. ${ }^{6}$

T1DE is associated with significant morbidity and mortality. ${ }^{7}$ Prolonged periods of high blood glucose resulting from insufficient insulin puts these individuals at profound risk of irreversible microvascular complications including retinopathy, peripheral neuropathy, renal disease and autonomic dysfunction. ${ }^{4,5}$ There are also short-term consequences including electrolyte imbalance, volume depletion, increased risk of yeast and bacterial infections, muscle atrophy and diabetic ketoacidosis. Over an 11-year study period, women with type 1 diabetes who restricted their insulin for fear of weight gain were 3.2 times more likely to die over the study period. ${ }^{7}$ They also died on average 13 years younger than those who didn't restrict insulin.

In light of the severity and impact of T1DE on those affected, designing care pathways and services to support these individuals is of great importance. One of the challenges is the identification of those affected by the condition. Healthcare professionals likely to encounter people with T1DE need to have confidence to engage in early detection, understand the increased risk prevalence and feel comfortable supporting, signposting and/or referring on to appropriate services.

Combined Pathway for Assessment and Support for the Syndrome of Insulin OmissioN - Type 1 Diabetes (ComPASSION) is one of two NHS England-funded pilot projects focusing on the development of services to help people with T1DE. It is currently running in Wessex, led by Dr Helen Partridge, consultant diabetologist at The Royal Bournemouth Hospital, and Dr Carla Figueiredo, consultant psychiatrist at Dorset HealthCare University NHS Foundation Trust. The aims of the ComPASSION project are to trial, test and evidence the impact of an integrated diabetes and mental health pathway for the assessment, referral and treatment of T1DE. The other NHS England-funded T1DE pilot project, led by Professor Khalida Ismail at King's College Hospital, has a focus on those with severe symptoms of T1DE. 
To understand the requirement for training amongst healthcare professionals in identifying, sign-posting and referring individuals with or at risk of T1DE, we designed a local crosssectional staff survey to help inform the ComPASSION project. Data from this will highlight the learning needs across settings (eg, primary care, secondary care and community services), and different healthcare professional groups. It will also establishhow healthcare professionals would like training to be delivered.

\section{Aims}

- To establish the awareness of T1DE amongst healthcare professionals locally across primary, community and acute healthcare settings who may come into contact with individuals affected by the condition.

- To determine the awareness, confidence and competencies of healthcare professionals in:

O being able to identify individuals with T1DE

o supporting people with T1DE

o knowing where to refer to for further support in managing T1DE

\section{Methods}

\section{Study design}

A cross-sectional, self-report, online survey using Survey Monkey was designed to gain data from a large cohort of healthcare professionals. Informed consent was recorded at the beginning of the electronically completed questionnaire. Responses were anonymous but participants were able to provide their email address to be contacted again at a later date for further studies. The study was approved by the Health Research Authority and Health and Care Research Wales: REC reference 19-HRA/4284; IRAS number 269186.

\section{Study participants}

Study participants included healthcare professionals in patient facing roles who may encounter individuals with type 1 diabetes and/or eating disorders as part of their day-to-day clinical practice. The study was limited to the geographical area of Wessex (Hampshire and Dorset) and was advertised to those working in acute trusts, primary care, community pharmacies, specialist diabetes services or specialist mental health settings including, but not limited to, eating disorder services.

\section{Inclusion criteria}

- Male or female, aged 18 years or above.

- Working as a healthcare professional in the geographical area of Wessex.

- Working in a patient-facing role where they may encounter people with type 1 diabetes and/or an eating disorder.

\section{Exclusion criteria}

- Those unwilling to participate.

\section{Recruitment}

Hospital trusts in Wessex advertised the study to potential staff participants using their intranets, newsletters and emails. The study was also promoted to general practitioners and community pharmacists through existing clinical networks including the Clinical Research Network for Wessex and the Local Pharmaceutical Committees, respectively. The initial recruitment period was 12 weeks but was extended to six months to facilitate recruitment. This population was chosen as it represents the catchment area of the pilot ComPASSION project.

\section{Survey questions}

Please see Appendix (online at www.bjd-abcd.com) for questions included in the survey.

\section{Results}

\section{Demographics of respondents}

848 healthcare professionals completed the survey in primary and secondary care across Dorset and Hampshire. There was a proportionate spread of respondents across the two counties (Hamphire $52 \%$, Dorset $48 \%$ ). A majority of respondents (724, $85.4 \%$ ) answered all questions on the survey. A breakdown of the respondent healthcare teams and their varying roles is shown in Table 1 and Table 2, respectively. A significant proportion of respondents were female ( $78.2 \%$ female vs $20.2 \%$ male). A small proportion (1.6\%) preferred not to disclose.

Table 1 Care teams of respondents (832/848 respondents (98\%) answered this question)

\begin{tabular}{ll}
\hline Care team & $\%$ \\
Primary care & $24.2 \%$ \\
Hospital-based & $53.1 \%$ \\
Diabetes Specialist Services & $12.3 \%$ \\
Community Pharmacy & $0.5 \%$ \\
Community Mental Health & $4.3 \%$ \\
Eating Disorders Services & $3.9 \%$ \\
Other community services (eg community rehab) & $1.7 \%$
\end{tabular}

Table 2 Healthcare roles of respondents (761/848 respondents (90\%) completed this questionnaire)

\begin{tabular}{ll}
\hline Healthcare role & Number (\%) \\
General practitioner & $131(15.5 \%)$ \\
Diabetes Speciality Doctor & $30(3.5 \%)$ \\
Doctor (other) & $44(5.2 \%)$ \\
Surgeon & $8(0.9 \%)$ \\
Psychiatrist & $4(0.5 \%)$ \\
Diabetes Speciality Nurse & $46(5.4 \%)$ \\
Mental Health Nurse & $33(3.9 \%)$ \\
Nurse (other) & $215(25.4 \%)$ \\
Psychologist/psychotherapist & $16(1.9 \%)$ \\
Dietitian & $24(2.8 \%)$ \\
Pharmacist & $27(3.2 \%)$ \\
Pharmacy technician & $6(0.7 \%)$ \\
Podiatrist & $8(0.9 \%)$ \\
Outreach/support worker & $11(1.3 \%)$ \\
Other allied healthcare professionals (eg, speech & $157(18.5 \%)$ \\
and language therapists, physiotherapists, midwives) & \\
Not disclosed & $88(10.4 \%)$
\end{tabular}


Figure 1. Confidence levels of respondents in recognising 'Type 1 Diabetes and Disordered Eating' (T1DE)

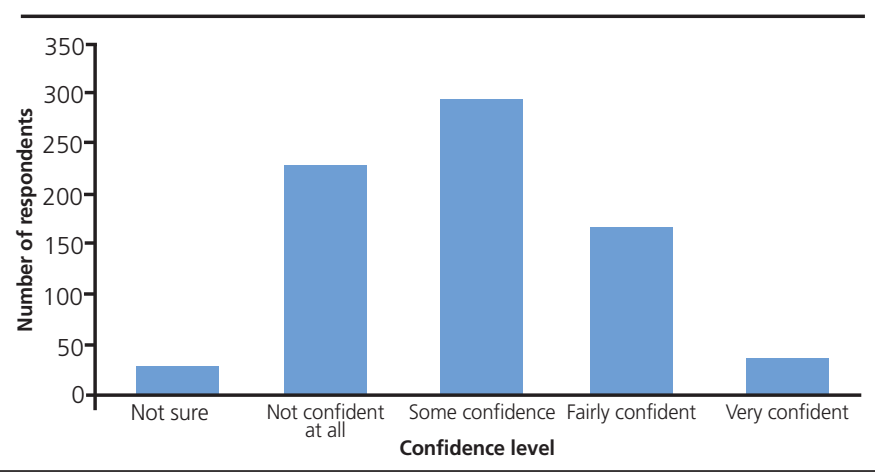

Figure 2. Confidence levels of specific healthcare professional groups in recognising 'Type 1 Diabetes and Disordered Eating' (T1DE)

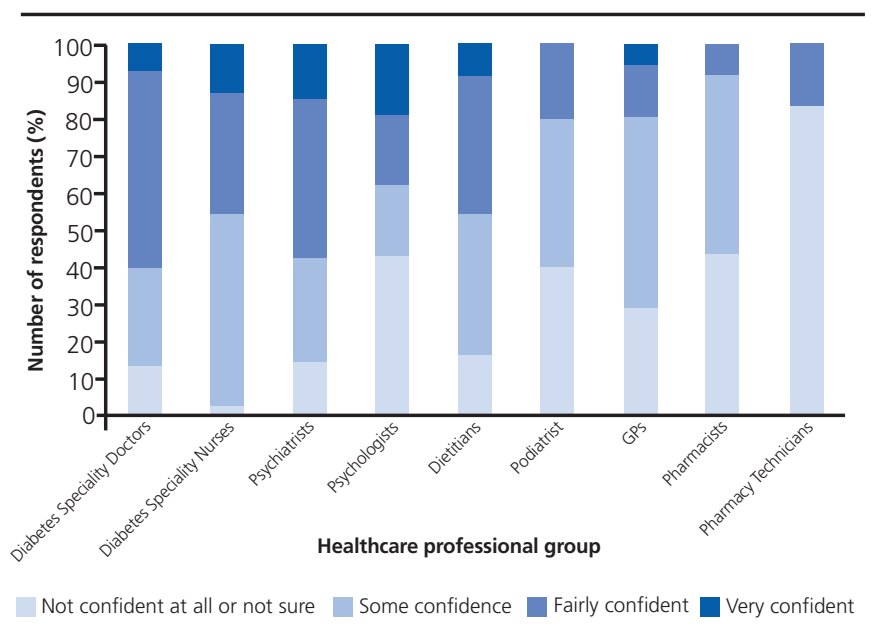

Current confidence levels around identifying an eating disorder in the context of type 1 diabetes

756 of the 848 (89\%) respondents answered this question. As depicted in Figure 1, the majority of respondents had low confidence in recognising T1DE. Confidence levels across a selection of healthcare professional groups are shown in Figure 2. Of the $39(5.2 \%)$ individuals who described themselves as 'very confident', only five (12.8\%) regularly manage people with T1DE.

What would make you consider a diagnosis of an eating disorder in the context of type 1 diabetes?

This was answered by 739 (87\%) respondents (Table 3 and Figure 3). Additional comments given by respondents that would prompt them to consider T1DE included those shown in Box 1.

\section{Have you ever managed or been involved in the care of someone with type 1 diabetes and an eating disorder before?}

Of the $735(87 \%)$ respondents who answered this question, a majority $(68.4 \%)$ had never managed or been involved in the
Table 3 Characteristics that would raise suspicion of T1DE (this was answered by 739 (87\%) respondents)

\begin{tabular}{ll}
\hline & $\begin{array}{l}\text { Number (\%) of respondents } \\
\text { selecting each option }\end{array}$ \\
\hline Elevated or rising glycaemic control & $388(52.6 \%)$ \\
Weight loss & $635(86 \%)$ \\
Recurrent diabetic ketoacidosis & $511(69.2 \%)$ \\
Restriction of insulin & $513(69.5 \%)$ \\
Not attending appointments & $472(64 \%)$ \\
Not picking up prescriptions & $498(67.5 \%)$ \\
The individual has disclosed it & $597(81 \%)$
\end{tabular}

Figure 3. Characteristics that would raise the suspicion of 'Type 1 Diabetes and Disordered Eating' (T1DE): frequency with which respondents selected each option

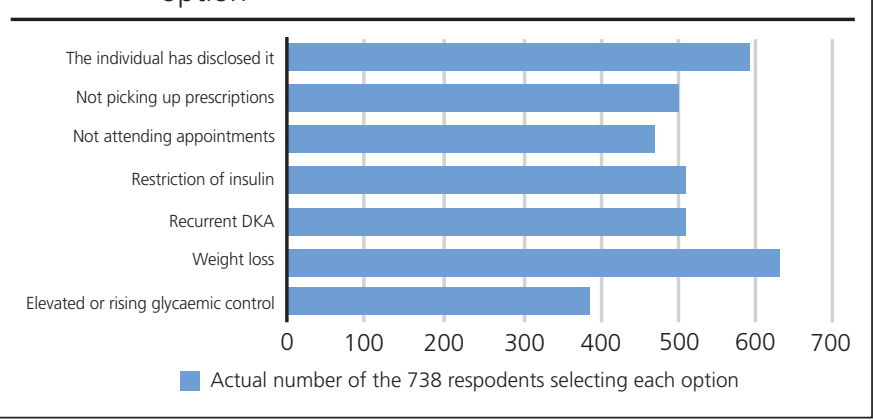

Box 1 Additional comments given by respondents that would prompt them to consider T1DE

- Family members had disclosed concerns

- Weight gain

- Binge eating behaviours, pre-occupation with food

- An unhealthy or driven relationship/pre-occupation with exercise or exercising without adjusting calories accordingly

- A history of other mental health conditions, poor selfconfidence, withdrawn

- The healthcare professional's previous experience with the condition and thus underlying intuition

- Disparity between what the person says they are taking and what their prescription pattern is

- Altered body perception, pre-occupation with body shape

- Reported 'good' blood glucose readings but in the presence of an elevated $\mathrm{HbA}_{1}$, physical appearance of the individual

care of someone with type 1 diabetes and disordered eating. Only $7.3 \%$ of the 232 people who had, do so regularly as part of their job.

\section{If you have managed or been involved in the care of} someone with an eating disorder in the context of type 1 diabetes, what went well or not so well?

Respondents who had been involved in the care pathway of someone with T1DE commented on what they felt had previously gone well or not so well, including the challenges and/or successes they encountered. 
Figure 4. Themes that were identified from the comments of respondents as being important in the care of those with an eating disorder in the context of type 1 diabetes

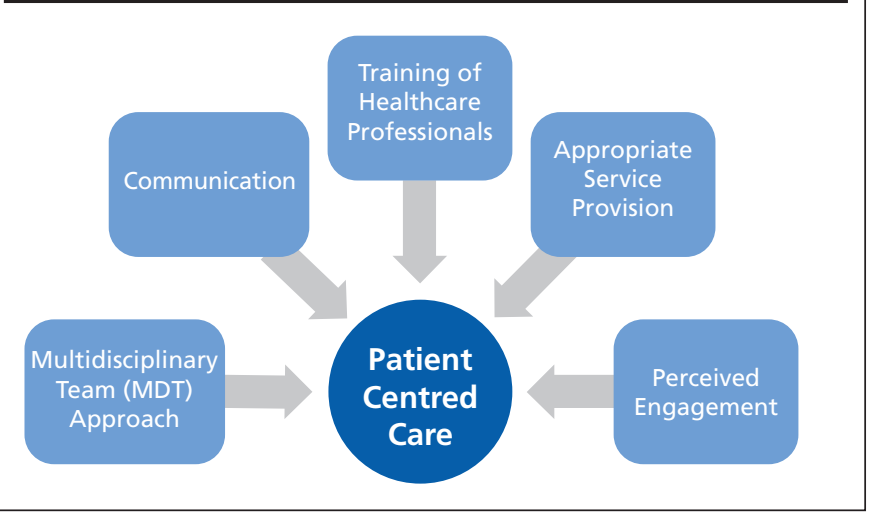

Many of the comments from respondents we attributed to describing a patient-centred approach. Five interdependent themes emerged which we felt fed into this (Figure 4).

\section{A Multidisciplinary Approach}

A multidisciplinary approach to care was described as important by many of the respondents, particularly to facilitate joined working, the sharing of decisions and management of risk (Box 2a). When a cohesive multidisciplinary team approach was lacking, this precipitated distress amongst those involved, particularly the patient (Box 2b).

Box 2 Quotes supporting a multidisciplinary approach to care

a)

- "having a full MDT including a dietitian to manage risk" Occupation Therapist, Eating Disorders service

- "Joint working with the diabetes team was extremely helpful, they joined an initial session and then we were able to contact them with regards to support in specific details related to the diabetes..." Psychologist

(b)

- "A lot of conflict about who had responsibility for administering the injections - staff or patient where patient would not take them. Patient unwell/ aggressive/upset about the process" Mental Health Trust Pharmacist

\section{Communication}

The different skill sets of healthcare professionals can be complementary and constructive in identifying and supporting someone with T1DE, but good communication with the individual and between services and healthcare professionals is required and can encourage continuity of care (Box 3a).

The use of appropriate language - particularly with the patient - was deemed important, in addition to being direct and transparent, helping to create a safe environment where everyone involved can be encouraged to be open and honest (Box 3b). Some healthcare professionals felt ill-equipped at using appropriate language or had identified colleagues using approaches they felt uncomfortable with (Box 3c). Difficulties stemmed from knowing how to communicate effectively whilst maintaining rapport when features of the illness led to a mismatch between objective measures and subjective accounts given by the individual (Box 3d).

Box 3 Quotes supporting the importance of communication

a)

- "Concerns were spotted by many health professionals but in different ways" Nurse

- "I feel awareness of this history ensures I can offer the most appropriate advice" Dietitian

- "No communication of outcome in some of the cases" Diabetes Speciality Nurse

- "Was always admitted under and seen by the same team so there was continuity" Diabetes Speciality Doctor

(b)

- "I realised that the motivational interviewing skills and language used when supporting people with diabetes or any long term condition provides a great foundation for communicating well with someone with an eating disorder" Diabetes Dietitian

- Patients felt comfortable to be open about their range of symptoms/coping mechanisms/progress" Healthcare Assistant

- "I asked directly the question about weight, which revealed the underlying issue" General Practitioner

- "Transparency and empathy went well" Consultant Gastroenterologist

(c)

- "Was very conscious of language and worried about saying the wrong thing" Diabetes Dietitian

- "Some of the team used scare tactics to address the problem" Diabetes Speciality Doctor

(d)

- " "Trying to maintain patient relationships during a time when you know the patient is telling you one thing but the prescription evidence and $\mathrm{HbA}_{1 \mathrm{c}}$ tells another story" Nurse Practitioner

\section{Training of healthcare professionals}

Training of healthcare professionals to facilitate recognition of T1DE, use of appropriate consulting skills and sign-posting to local services were all described by respondents as a fundamental part of care. Most respondents felt they didn't have the relevant expertise and expressed a wish for this to be addressed (Box 4a).

Healthcare professionals attributed blame to their lack of knowledge as a contributing factor to missed or late diagnoses of the condition, inappropriate management or unease when supporting someone with the condition (Box 4b).

A lack of recognition of the condition as being a unique entity, distinct from either type 1 diabetes or an eating disorder in isolation was highlighted (Box 4c). Where possible, healthcare professionals want to be able to attempt to understand the underlying drivers to the behaviours they were seeing to be able to offer a more patient-centred approach (Box 4d). 


\section{Box 4 Quotes on training of healthcare professionals}

a)

- "I wish that I had had education at the time so that I could have recognised this sooner, or known where to get

(b) information to support the patient" Renal Nurse

- "... not recognised by colleagues as an issue" Nurse

- "I think with hindsight this was probably the diagnosis ..." General Practitioner

- "difficult to get the patient to co-operate, I needed more knowledge" Midwife

- "If I'm honest, I found the subject difficult to broach with her as I felt I didn't have the expertise to support her ..." Diabetes Speciality Nurse

- "Lack of skills (mine) in determining whether this was disordered eating or an eating disorder, where to go to get help/referral pathways, knowledge of the approach taken by the eating disorders team (which was different to our usual approach in that it was more prescriptive than I am used to)" Diabetes Specialist Nurse

(c)

- "Identifying the issues were easier than getting the follow-up support for patients as not currently recognised as an eating disorder" Diabetes Specialist Nurse

(d)

- "Hard to decide what this is due to, and some may be trying to keep weight off, some may want to have control over some part of the situation, and others just want to have a normal life and may be annoyed at their diagnosis and just not want to engage" Diabetes Specialist Nurse

\section{Appropriate service provision}

The need for a clear patient pathway with adequate availability of appropriate services was described. Respondents articulated difficulties in accessing support, particularly mental health support (Box 5a). Respondents felt that existing services are not always appropriate, joined up or equipped to manage (Box 5b).

\section{Box 5 Quotes on appropriate service provision}

a)

- "Very difficult to find specialist psychology/psychiatry services " Diabetes Speciality Doctor

(b)

- " "... many of the Eating Disorder Units were not willing to accept her based on her diagnosis of diabetes as they did not feel confident managing the insulin on the ward" Psychologist

- $\quad$ "Lack of integration of available support between Diabetes service and MH support" General Practitioner

\section{Perceived engagement}

A challenge raised by healthcare professionals included managing the different aspects of the two conditions which have a significant impact on one another (Box 6a). What was termed 'disengagement' and the behaviour of non-attendance at appointments were recognised as issues when trying to offer best care (Box 6b).

How confident do you feel talking to someone you think may have an eating disorder and type 1 diabetes?

In support of the comments from the previous question, a
Box 6 Quotes on particular challenges faced and perceived engagement

a)

- "managing the restrictions she imposed on herself, as well as the fact she had developed other complications from mismanaging"Dietitian

- "hard to avoid hypos/DKA with intermittent food intake and insulin dosing" Hospital Pharmacist

- "frustrating - dealing with the aftermath of years of high blood glucose and now partially blind, neuropathy of limbs and receiving haemofiltration" Critical Care Nurse

(b)

- "... lots of DNA making it hard to establish a relationship at the start" Diabetes Dietitian

majority of 735 respondents felt they had 'no confidence at all' (32\%) or only 'some confidence' (39.5\%); $20 \%$ were 'fairly confident', $5.7 \%$ 'very confident' and $2.9 \%$ were 'not sure'.

\section{How confident do you feel about offering advice and} support around insulin/diabetes/weight management for someone with type 1 diabetes and an eating disorder? Most of the 730 responding healthcare professionals are 'not confident at all' (54.5\%) or class themselves as 'somewhat confident' (27.4\%) or 'not sure' (3\%) in offering advice and support around insulin/diabetes and weight management. Of those that felt more confident (12.5\% 'fairly confident', $2.6 \%$ 'very confident'), there was no clear association with their underlying job role.

Thirty out of 76 diabetes specialist doctors/nurses completing the survey (40\%) classified themselves as 'fairly confident' or 'very confident' in offering advice and support around diabetes, insulin and weight management. In comparison, this was the case for one out of four psychiatrists (25\%), eight out of 131 general practitioners (6\%) and six out of 24 dietitians (25\%).

\section{Education on type 1 diabetes and eating disorders}

$66.6 \%$ of the 732 respondents had never received any education on T1DE. The varying modes of education delivery and their frequency reported by the $33.4 \%$ of respondents who had is shown in Figure 5.

Figure 5. Modes of education delivery and their frequency

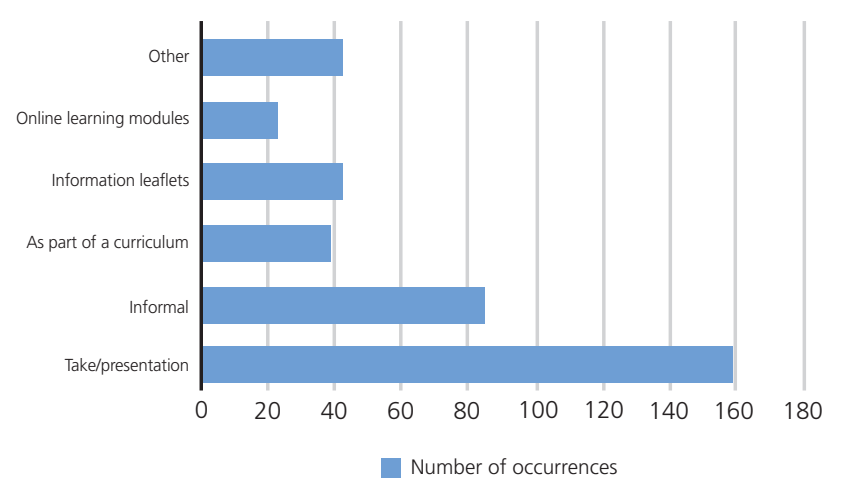


Figure 6. Where respondents would go for additional support when managing someone with an eating disorder in the context of type 1 diabetes

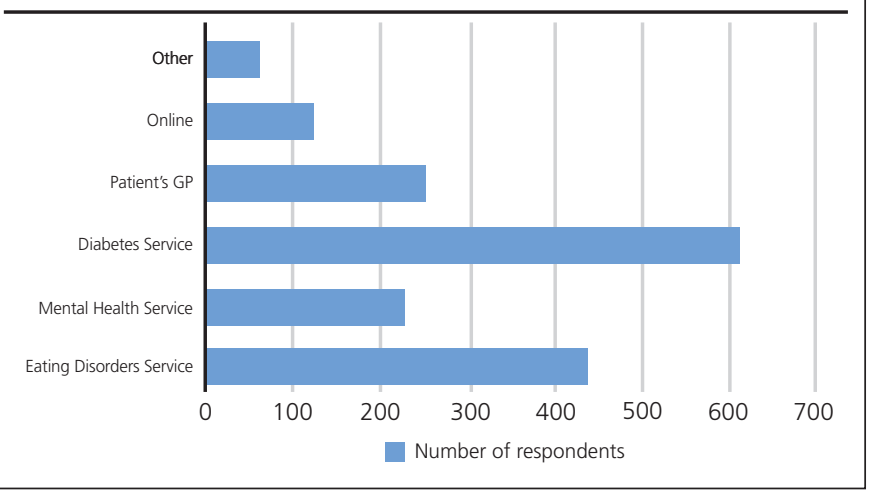

Other forms of education described by respondents included personal reading/study, webinars, a television documentary and personal experiences when they themselves or a family or friend had been affected.

How confident do you feel knowing where to turn for additional help/support when you have identified someone with type 1 diabetes that you are concerned may have an eating disorder?

123 of the 731 people who completed this question (16.8\%) were 'unsure' or 'don't feel confident' knowing where to go to seek additional support when concerned about someone they feel may have T1DE. The services to which people would go are shown in Figure 6. 5.2\% of people would consult all of the options listed.

When invited to offer additional comments, respondents suggested that they would additionally consider contacting their senior, a dietitian, team psychologist, diabetes counsellor, general practitioner with a specialist interest in diabetes or a diabetes pharmacist.

\section{Further education on eating disorders in type 1 diabetes and the services available locally}

A predominance of the 730 respondents (71\%) expressed an interest in receiving further education on T1DE and the services available locally to them. $6.6 \%$ were not interested and the remainder selected 'maybe' as an option.

When asked about mode of delivery, a majority (64\%) would like to have further education take place in a face-to-face format, with $28 \%$ having selected this as their only preference. Additional comments identified that respondents would want this to take place locally at the work place or for study time to be allocated to it (eg, a study day), ideally with a certificate for Continued Professional Development (CPD). Just over half of respondents (52\%) would be happy with an online format including online webinars and tutorials, with $15.8 \%$ choosing this as their only preference. A significant number would also be comfortable with an information pack (41.5\%), but only 9.5\% would be happy for an information pack in isolation. $15.5 \%$ selected all three options.

\section{Additional closing comments}

The survey was well received and felt to be a subject worth better understanding by a majority, but for some the subject was felt to be very specialised (Box 7a). It highlighted a knowledge gap for many but an appetite for learning more (Box 7b). An interest in a patient pathway and information and clarity on local services was expressed (Box 7c). Some additional considerations and thoughts were also highlighted in the respondents' comments (Box 7d).

\section{Box 7 Additional comments made by respondents}

a)

- "little explored topic, worth investigating" General Practitioner

- "I am thankful that a spotlight is being shone on this serious under-recognised hugely complex issue and hope that we can all learn how best to support PWD and insulin omission/eating disorders." - Diabetes Speciality Nurse

- "I am not entirely sure how relevant this is to my day-to-day practice (eating disorders, yes, but the combination is very specialised ...)" Consultant

(b)

- "I am not knowledgeable about this condition. This survey has highlighted this." Healthcare Assistant

- "This is a great initiative ... badly needed for those of us who do not work in specialist services and only come across these patients occasionally" Physiotherapist

- "Was not even aware of such an issue. More education please ..." General Nurse

- "Any education around this would be good" Midwife

- "GPs need some sort of training in this so their awareness improves. These types of patients, especially the ones highly skilled at glycaemic management, can fly under the radar and only the GPs stand to be able to pick up the issues" Diabetes Specialist

- "Have worked in an eating disorders service previously but not experienced the combination with type 1 diabetes so

(c) awareness of this is poor" Psychologist/Psychotherapist

- "As a general surgeon with an interest in pancreatic disease, I would value a simple sign-posting/ referral pathway for suspected ED/type 1 DM patients"

- "I am pleased this study is being completed as it is an area that needs investment and support to develop a clear nationwide NHS pathway for this complex condition to ensure those who suffer with diabulimia get the right support at the right time." Psychiatrist

(d)

- "Hadn't thought about this prior to survey" Anaesthetist

- "I ... would always seek advice from my diabetes colleagues re these patients. I am concerned that sometimes DKA patients get admitted to critical care and then sent home from critical care without a huge amount of diabetes team input and these patients may slip through the net." Critical Care Consultant

- "I am happy speaking with and supporting patients with EDs. I think the area I lack is taking the next step into implementing strategies which actually help them" Dietitian

- "Most patients with newly diagnosed type 1 DM are being started on insulin and subsequently monitored by hospitalbased specialist diabetes nurses, hence most GPs will lack the training and experience to both manage type $1 \mathrm{DM}$ and to recognise the signs and symptoms of 'diabulimia'." General Practitioner

- "I rarely see young type 1 diabetics. They are most often seen at a diabetic specialist clinic and they don't seem to feel they need anything extra from their GP" General Practitioner 


\section{Discussion}

\section{Main findings}

The results from this survey have provided an insight into the underlying awareness and understanding of T1DE amongst healthcare professionals and concerns they have relating to this topic. Interestingly, a large majority of respondents were female. It could be speculated that males prefer not to complete surveys or are less interested in the subject area, or perhaps females make up the greater proportion of the healthcare professional pool amongst which the study was promoted. From the data collected, it is not possible to attribute an explanation to this finding.

A significant majority of respondents had no or only limited confidence in recognising T1DE. This was true across different speciality groups and healthcare settings represented in this study. A majority had never previously managed or knowingly been involved in the care of someone with T1DE.

Comments from a variety of participating healthcare professionals have highlighted how people with T1DE may only get picked up when at crisis point, presenting with complications including diabetic ketoacidosis and foot problems amongst others, or flagged as having missed their annual diabetes reviews. Increasing recognition and appreciation of the condition amongst healthcare professionals may facilitate earlier intervention to support affected individuals.

'Weight loss' and 'disclosure from the individual' were the two most selected identifying features for T1DE selected by the participants. Both features are unreliable - people with T1DE rarely disclose it and T1DE is less frequently associated with the same degree of weight loss as is typically seen in anorexia nervosa. 'Elevated or rising glycaemic control' was the least frequently selected option and 'restriction of insulin' was considered a feature by $69.7 \%$ of healthcare professionals. Both of these are suggestive of T1DE in association with an individual's concerns about their body weight or body image.

As published work suggests, the differing terminology used to describe the behaviour of insulin omission in type 1 diabetes for fear of weight gain can lead to confusion. The condition is not currently recognised as a unique entity in psychiatric classifications. In this context, low confidence in recognising and managing T1DE may explain why difficulties arise when trying to find specialist care to support those affected.

There was an impression and concern that limited services are available to support people with T1DE and that healthcare professionals were not confident about who or where to refer for support with someone they are concerned about.

Germane to the work of the ComPASSION project, healthcare professionals expressed an interest in knowing more about services available locally and where to sign post to. An appreciation for the importance of appropriate language was expressed alongside an interest in having training on suitable consulting skills.

\section{Strengths and limitations of this study}

To our knowledge, this is the first study of its kind assessing healthcare professional awareness of T1DE, however caution must be taken when drawing conclusions from some of the findings. There was not an equal or proportionate representation of each healthcare group. Some questions were also not answered by every respondent, which will have had an impact on the quoted figures. The survey was open for approximately 6 months (1 October 2019 to 31 March 2020), but due to participating trusts and organisations advertising it internally using differing strategies, this may explain why some were better represented than others. With the survey only being available and accessible to respondents online, this may have introduced bias, although it can be inherently assumed that most healthcare professionals have access to this technology.

The study population and geographical area was chosen as it represents the catchment area of the Wessex led ComPASSION pilot project which it was designed to inform. Whilst it is not possible to generalise the findings to other populations, the counties of Dorset and Hampshire are diverse. They include major cities like Southampton and Portsmouth and a large rural geography. In contrast to some regions, however, according to the last Census in 2011, Wessex has a greater proportion of people aged 65 years and over (Dorset 28\%, Hampshire 17.1\%) compared with the national average (18\%).8,9 They also have lower levels of black and minority ethnic residents (Dorset $4.4 \%$, Hampshire $8.2 \%$ ) compared with the country as a whole (19.5\%). In terms of deprivation, 10 out of a total of 219 areas in Dorset are within the top $20 \%$ most deprived nationally for multiple deprivation. In Hampshire, 40 out of 832 areas are in this category.,10

The ComPASSION project has been publicised in Wessex. As the survey was carried out in this area, there is the possibility that the number of people who are aware and confident of T1DE may be over-represented compared with healthcare professionals working in other areas.

Finally, as with all surveys, individuals invited to take part who choose not to respond are a unique group in their own right. We are unable to comment on the reasons individuals chose not to participate or draw conclusions on their appreciation of the subject matter. The same applies for those who completed the survey but skipped various questions.

\section{Implications for future research, policy and practice} The findings from this study will contribute to the ComPASSION project and facilitate the development of initiatives to raise awareness of T1DE and improve the knowledge and skills set of those working in settings where they are likely to encounter individuals with the condition. The results suggest that generic initiatives are important to raise global awareness, but more tailored approaches are required to meet the specific needs of differing staff groups and/or settings. As with other forms of diabetes training, education on type 1 diabetes and disordered eating could be delivered in levels/tiers. Level 1 could focus on raising awareness of the condition in the vast majority of healthcare professionals. Progressing levels would aim to deliver increasing quantity and depth of training for those likely to encounter and be involved in the management of the condition.

In terms of education delivery, face-to-face teaching was 


\section{Key messages}

- People with type 1 diabetes are at increased risk of eating disorders

- 'Diabulimia', 'Syndrome of Insulin Omission' (SIO) and 'Type 1 Diabetes and Disordered Eating' (T1DE) are all terms used to describe the practice of reducing or omitting insulin in order to lose weight. This behaviour is not a formally recognised medical or psychiatric condition

- To develop services to support individuals with T1DE, it is important to first understand the current levels of healthcare professional knowledge and awareness of the condition so that relevant training can be delivered

- Healthcare professionals have low confidence in their ability to recognise T1DE and how to best support affected individuals, but expressed an interest in learning more

- Comments from respondents on factors they felt were important in the care of those with T1DE fell into four broad themes: communication, a multidisciplinary approach, adequate healthcare professional training and appropriate service provision

popular but the flexibility of online methods was also appreciated. Approved study time to complete the training was voiced as being important. To encourage uptake, accreditation of any learning material should be considered.

\section{Conclusions}

T1DE is associated with significant increased morbidity and mortality. Healthcare professional confidence in recognising and supporting an individual whom they suspect may have an eating disorder in the context of type 1 diabetes is low across Dorset and Hampshire. This survey supports the work currently being done as part of the ComPASSION project, developing a patient pathway for those affected by T1DE. Healthcare professionals who are in roles where they may encounter someone affected want to know how to confidently recognise the condition, how to best support individuals and, importantly, how to access the services available to them locally. Where possible, this teaching should be relevant to the role and healthcare setting of the individual, accessible and supported by employers.

\section{Conflict of interest None to declare.}

Funding No funding was received for this questionnaire study.

\section{References}

1. Jones JM, Lawson ML, Daneman D, Olmsted MP, Rodin G. Eating disorders in adolescent females with and without type 1 diabetes: cross sectional study. BMJ 2000;320(7249):1563-6.

2. Colton P, Olmsted M, Daneman D, Rydall A, Rodin G. Disturbed eating behavior and eating disorders in preteen and early teenage girls with type 1 diabetes. Diabetes Care 2004;27(7):1654-9. https://doi.org/10.2337/diacare.27.7.1654

3. Colton PA, Olmsted MP, Daneman D, et al. Eating disorders in girls and women with type 1 diabetes: a longitudinal study of prevalence, onset, remission, and recurrence. Diabetes Care 2015;38(7):1212-17. https://doi.org/10.2337/dc14-2646

4. Bryden KS, Neil A, Mayou RA, Peveler RC, Fairburn CG, Dunger DB. Eating habits, body weight, and insulin misuse. A longitudinal study of teenagers and young adults with type 1 diabetes. Diabetes Care 1999;22(12):195660. https://doi.org/10.2337/diacare.22.12.1956

5. Rydall AC, Rodin GM, Olmsted MP, Devenyi RG, Daneman D. Disordered eating behavior and microvascular complications in young women with insulin-dependent diabetes mellitus. N Engl J Med 1997;336(26):1849-54. https://doi.org/10.1056/NEJM199706263362601

6. Mannucci E, Rotella F, Ricca V, Moretti S, Placidi GF, Rotella CM. Eating disorders in patients with type 1 diabetes: a meta-analysis. J Endocrinol Investig 2005;28(5):417-9. https://doi.org/10.1007/BF03347221

7. Goebel-Fabbri AE, Fikkan J, Franko DL, Pearson K, Anderson BJ, Weinger K. Insulin restriction and associated morbidity and mortality in women with type 1 diabetes. Diabetes Care 2008;31(3):415-19. https://doi.org/ $10.2337 / \mathrm{dc} 07-2026$

8. Hampshire County Council. 2011 Census: Equality and Diversity Profile- Hampshire County Council Area 2013. Available from: https://documents.hants.gov.uk/EqualityandDiversityFactsheet-HampshireCountyCouncilArea.pdf

9. Dorset Council. Topic Data. 2020. Available from: https://apps. geowessex.com/insights/Topics

10. Hampshire County Council. The 2019 Index of Multiple Deprivation. 2019 Available from: https://documents.hants.gov.uk/Economy/IndexofMultipleDeprivation.pdf 
Appendix 1. Survey questions

1) Which county do you work in?

(Dorset, Hampshire, Isle of Wight, other)

2) What type of team do you currently work in?

(Community mental health, eating disorders service, diabetes specialist services, primary care, community pharmacy, acute setting, other)

3) What type of role do you currently work in?

(Psychiatrist, psychologist, mental health nurse, diabetes specialist doctor, diabetes specialist nurse, general practitioner, pharmacist, pharmacy technician, dietitian, other)

4) What gender do you identify as?

(Male, female, prefer not to say)

5) How confident do you feel recognising the combination of type 1 diabetes and an eating disorder?

(Not confident, some confidence, fairly confident, very confident, not sure)

6) What would make/has made you consider a diagnosis of an eating disorder in type 1 diabetes? (Please tick all that apply) (Elevated or rising $\mathrm{HbA1c}$, recurrent diabetic ketoacidosis (DKA), weight loss, restriction of insulin, not collecting prescriptions for insulin, not attending appointments, the individual has disclosed it, other

7a) Have you ever managed or been involved in the care of someone with type 1 diabetes and an eating disorder before? (Never, a couple of times, a handful of times)

7b) If yes, please provide details on what you felt went well or not so well.

8) How confident do you feel talking to someone you think may have an eating disorder and type 1 diabetes?

(Not confident, some confidence, fairly confident, very confident, not sure)

9) How confident do you feel about offering advice and support around insulin/ diabetes/weight management for someone with type 1 diabetes and an eating disorder?

(Not confident, some confidence, fairly confident, very confident, not sure)

10a) Have you ever had any education on type 1 diabetes and eating disorders?

(No, Some, Yes)

10b) If 'some' or 'yes,' how was it delivered?

(Talk/presentation, informal teaching, information leaflet/booklets, as part of speciality/training curriculum, online learning module, other)

11) How confident do you feel about knowing where to turn for additional help/ support?

(Not confident, some confidence, fairly confident, very confident, not sure)

12) Where would you go for additional support?

(Eating disorders services, mental health services, diabetes services, patient's GP, online, other)

13a) Would you be interested in participating in further education on eating disorders in type 1 diabetes and the services available locally? (Yes/No)

13b) If 'yes', how would you like this to be delivered?

(Face-to-face, information pack, online webinar or module, other)

14) Please provide any additional comments below: 